\title{
Komplexe Organisation und organisierte Komplexität. Die Pädagogik partizipativer Organisationsgestaltung im Zeitalter des Populismus
}

\author{
Sebastian Manhart • Thomas Wendt
}

Eingegangen: 7. Juli 2020 / Überarbeitet: 24. September 2020 / Angenommen: 19. Oktober 2020 / Online publiziert: 9. November 2020

(C) Der/die Autor(en) 2020

Zusammenfassung Organisationen sind die zentralen Komplexitätsgeneratoren der modernen Gesellschaft. Sie regulieren und steigern die Möglichkeiten gleichzeitiger Handlungsvollzüge immer weiter. Der Beitrag konzipiert Populismus als gesellschaftsweit verbreitete Form der Kritik an dieser organisierten Komplexität und grenzt ihn systematisch von Fundamentalismen und Verschwörungstheorien als weiteren wichtigen komplexitätsreduzierenden Deutungen der Moderne ab. Die Strukturlogik von Organisationen und ihre Rolle in der Gesellschaft wird im Verhältnis zu den gesellschaftlichen Ansprüchen auf individuelle Teilhabe analysiert. Die pädagogischen Potenziale partizipativer Organisationsgestaltung werden im Blick darauf diskutiert, dass dieser Modus systematisch erzeugter Komplexität sich damit selbst populistischer Kritik aussetzt.

Schlüsselwörter Organisation · Komplexität · Populismus · Partizipation · Bildung

Prof. Dr. S. Manhart

Universität der Bundeswehr München, München, Deutschland

E-Mail: sebastian.manhart@unibw.de

Dr. T. Wendt $(\bowtie)$

Universität Trier, Trier, Deutschland

E-Mail: wendtth@uni-trier.de 


\title{
Complex organization and organized complexity. Educational Aspects of Participatory Organizational Structuring in the Age of Populism
}

\begin{abstract}
Organizations function as the central generators of complexity in modern society by regulating and constantly increasing the opportunities for simultaneous action. The paper conceives populism as a socially widespread form of criticism of this organized complexity and systematically differentiates it from fundamentalisms and conspiracy theories as important complexity reducing interpretations of modernity. The structural logic of organizations and their role in society is analyzed in relation to society's demands for individual participation. The educational potentials of participatory organizational structures are discussed in view of the fact that this mode of systematically generated complexity exposes itself to populist criticism.
\end{abstract}

Keywords Organization $\cdot$ Complexity $\cdot$ Populism $\cdot$ Participation $\cdot$ Bildung

\section{Einleitung}

An Zuschreibungen der Art, etwas sei populistisch, herrscht derzeit kein Mangel. Weniger klar ist, was diese Zuschreibungen genau meinen (vgl. Vehrkamp und Merkel 2020). Vermutlich besteht hier ein Zusammenhang. Es scheint umso leichter, jemanden als Populisten zu bezeichnen, je weniger klar ist, was das bedeutet. Eine Erwachsenenbildung, die an zielgruppenadäquaten pädagogischen Strategien interessiert ist, kann es hierbei nicht belassen. Im Folgenden wird daher Populismus gesellschaftstheoretisch näher bestimmt. Eine sozialräumliche Ausweitung der Perspektive ergänzt und präzisiert die schon vorhandenen zeitlichen (Rosanvallon 2016, 2017; Raphael 2017), politischen (Wielenga und Hartleb 2011; Priester 2012; Diendorfer und Sandner 2017; Möllers und Merzel 2018) wie sozialstrukturell-ökonomischen (Manow 2018) Herleitungen des Populismus.

Populismus ist weder ein ganz neues noch ein ausschließlich politisches Phänomen. Es handelt sich um eine historisch gewachsene Denk-, Wahrnehmungsund Kommunikationsform, die gesellschaftsweit auftritt, weil sich ihre kritische Komponente an grundlegenden Strukturen der modernen Gesellschaft, nämlich an Organisationen entzündet. Deren intransparente Binnenkomplexität liefert permanent Anlässe für Kritik. Über die Allgegenwart von Organisationen vermittelt sich gesellschaftsweit die Erfahrung, dass externe Instanzen, wie z.B. die Legislative, die eigenlogische organisationale Dynamik kaum bzw. nicht zielgenau beeinflussen können. In einem kontroll- und steuerungsaffinen Verständnis von Gesellschaft wird dadurch nicht nur die Legislative delegitimiert, sondern auch die Partizipation an der Politik mittels Wahlen, Parteien, Ehrenämtern etc. Da sowohl politische Willensbildung, Repräsentation als auch die Umsetzung von Maßnahmen durch Exekutive und Judikative auf Organisationen angewiesen ist, trifft diese Kritik die Demokratie ins Mark. Neben den zahllosen Fehlschlägen einer staatlichen Kontrolle anderer gesellschaftlicher Subsysteme führt Politik regelmäßig an sich selber vor, worauf populistische Einlassungen reagieren. In der Dauerreflexion massenmedialer Realitätskonstruktion (Luhmann 1996) wird diese Kontroll- und Steuerungslücke 
permanent thematisiert. Das setzt traditionelle Formen politischer Partizipation und Repräsentation (Rosanvallon 2016; Decker 2017; Mouffe 2018) unter erheblichen Begründungsdruck.

Populistische Kritik an der Politik als Hinweis auf eine wesentlich politische Ursache des Populismus zu nehmen, stellt aber eine Verkürzung dar, die auch pädagogische Handlungsmöglichkeiten unnötig verengt. Denn Organisationen und institutionelle Regelungsgeflechte entziehen sich überall einer direktiven, externen wie internen Steuerung. Organisierte Zusammenhänge werden deshalb auch überall gern als unnötige Verkomplizierung und Beschränkung, als Überregulation der Gesellschaft denunziert. Populistische Kommunikation findet sich im Feld der Sozialen Hilfen, im Medizinsystem, im Sport, in der Wissenschaft, der Religion (Lesch 2017) und auch gegenüber jeder Form organisierter Pädagogik. Die Politisierung solcher Kritik durch pauschale Adressierung von Handlungsbedarf an die Politik ändert nichts daran, dass die meisten populistischen Kritiken und Narrative (Rosanvallon 2017; Müller und Precht 2019) weder in ihrer Form noch in ihrem Inhalt genuin politisch sind. Ein gesellschaftstheoretischer Phänomenzuschnitt erweitert daher die Interventionschancen der Erwachsenenbildung. Denn Populismus kann in allen Feldern der Erwachsenenbildung bearbeitet werden. Die Möglichkeiten und Grenzen von Organisationen können z.B. in der beruflichen, betrieblichen Bildung praktisch demonstriert und differenziert reflektiert werden. Das gilt nicht zuletzt für das eigene Beispiel einer jeden Form organisierter Pädagogik.

Für eine regelaverse populistische Kritik sind Organisationen die wichtigsten Generatoren, denn im Unterschied zu anonymen institutionellen oder funktionalen Strukturen sind sie leicht adressierbar. In einem ersten Schritt werden daher Rolle und Eigenlogik von Organisationen in der modernen Gesellschaft skizziert (2). Die historische Diskussion um den demokratisch verfassten Staat zeigt ihn als ein traditionelles Objekt populistischer Kritik (3). Obwohl es sich um sehr unterschiedliche Typen des Denkens handelt, werden Populismus, Verschwörungstheorien und Fundamentalismen politisch oft vermischt. Das diffuse Konstrukt Populismus (Hellmuth 2019) erhält durch eine sorgfältige Abgrenzung dieser Typen mehr Kontur (4). Die Tendenz zur Vermischung zeigt, dass eine politische Bestimmung des Begriffs „Populismus“ nicht ausreichend ist. Die Schwierigkeiten einer als politisches Gegenmittel propagierten Demokratisierung der Demokratie (Manow 2018) verdeutlichen dies exemplarisch. Verstärkte politische Partizipation und individuelle Teilhabe sind ohne eine Steigerung organisierter Komplexität nicht zu haben. Damit verschärft die politische Lösungsstrategie zugleich das Problem. Darauf kann die Erwachsenenbildung nicht nur andere vorbereiten, sondern auch sich selbst (5).

\section{Organisation in Gesellschaft}

Die moderne Gesellschaft ist auf die Ordnungsleistungen von Organisationen angewiesen. Mittels Organisationen werden Arbeitsplätze vorgehalten, Konsumgelegenheiten eröffnet, pädagogische Anforderungen verstetigt oder partizipative Ansprüche durchgesetzt. Unternehmen und staatliche Einrichtungen bieten Möglichkeiten und distribuieren Zugangschancen zur partiellen Entfaltung individueller Lebensentwür- 
fe. Gleichzeitig fungieren Organisationen als zentrale Komplexitätsgeneratoren der Gesellschaft, indem sie durch Prozesse der Strukturbildung gleichzeitige Handlungsoptionen und -vollzüge immer weiter vermehren (Wendt 2019). Diese Vermehrung von Möglichkeiten hat jedoch die Ausweitung von Einschränkungen zur Voraussetzung (Luhmann 2000b; Nassehi 2011, S. 193-237; Wendt 2016). Organisation, Regulation und Komplexität sind Synonyme. Die funktional differenzierte Gesellschaft erzeugt in sich eine Vielfalt undurchschaubarer Regulationsgeflechte, auf die sowohl pädagogische Konzepte (Manhart 2018) als auch populistische Angebote reagieren, indem sie Komplexität in leicht verständliche Formen gießen (Nassehi 2017). Dass beide Formen der Komplexitätsreduktion nicht identisch sind, darauf kommt es pädagogisch an.

Die gesellschaftliche Komplexität gründet in einem Prinzip, das die Reduktion von Komplexität zum eigenen Komplexitätsaufbau nutzt: Organisation. Die Gesellschaft der Gegenwart ist eine Organisationsgesellschaft. Organisationen ermöglichen die Parallelisierung einer Vielzahl gleichzeitiger Handlungsvollzüge (Wendt 2019), sie ermöglichen Kommunikation unter Abwesenden (Luhmann 2000b; Manhart 2009) und sie tun auch dies gleichzeitig, weil ihr Wirkprinzip auf der Verknüpfung von Motivgeneralisierung und Verhaltensspezifikation beruht (Luhmann 1975a). Unabhängig von der jeweiligen Motivlage der beteiligten Subjekte werden Handlungsvorgaben und -erwartungen an diese adressiert, um Tätigkeiten, die auf einen oder mehrere Zwecke ausgerichtet sind, parallel wie sequentiell zu koordinieren. Organisationen abstrahieren von individuellen Eigenheiten, um sich aus arbeitsteiligen Prozessschritten als Gesamtzusammenhang zu integrieren. Das Adressieren spezifischer Erwartungen und die Durchsetzung von Handlungsimperativen grenzt Organisationen von ihrer Umwelt ab (Baecker 2001). Auf diese Weise führen Organisationen zu einer erheblichen Ausweitung und Spezifizierung gesellschaftlicher Arbeitsteilung. Sie sind daher Lösung und Problemgenerator im Umgang mit Komplexität. Hinter sozialen Zwängen und Begrenzungen stecken oft Effekte organisationaler Strukturbildung. Organisation heißt, die Logik der Regulierung und geordneter Verfahren individueller Spontanität und Emotionalität vorzuziehen. Dies zu lernen, setzt nicht nur mindestens zwölf Schuljahre und unzählige normierte Freizeitangebote voraus, sondern gelingt auch nicht ohne individualisierende Abweichung. Gerade ihre Komplexität macht Organisationen daher zu zentralen pädagogischen Gebilden, die nicht nur Erziehung, sondern auch Bildung ermöglichen (Manhart 2009; Manhart und Wendt 2020; Wendt 2020).

Diese Pädagogik der Organisation entfaltet sich auch in demokratischen Systemen entlang des Spannungsverhältnisses zwischen Regulation und Partizipation. Demokratie bedeutet nicht nur eine bestimmte Organisation der Regierung, sondern sie setzt auch zahlreiche Organisationen der politischen Willensbildung, exekutiver wie legislativer Funktionen und ihrer Verwaltung voraus. Demokratische Teilhabe und die Gewährung gleicher Zugangschancen sind aber keine typischen Strukturprinzipien von Organisationen, vielmehr erzeugen sie legitime Ungleichheiten. Die organisationale Logik einer formal regulierten Mitgliedschaft legitimiert den Einschluss einer Minderheit bestimmter Personen zuungunsten einer Mehrheit, die ausgeschlossen bleibt. Das gilt auch intern: Organisationen beruhen auf hierarchischen Verhältnissen der Über- und Unterordnung und erst die, andere Mitglieder ausgren- 
zende, Regelung von Zuständigkeiten und Kommunikationswegen erzeugt stabile organisationale Muster. Vertikale Koordination und horizontale Differenzierung behindern Prozesse des gleichrangigen Austauschs ebenso wie Organisationen individuelle Partizipation zwar unter bestimmten Bedingungen ermöglichen, aber genau deshalb in fast allen anderen Fällen verhindern. So gesehen, fungieren Organisationen als gesellschaftliche Ungleichheitsgeneratoren, da allgemeine Gleichheitsund Teilhabeansprüche und deren organisierte Verwirklichung einander immer auch zuwiderlaufen (Luhmann 1994).

Schon aus diesem Grund sind Organisationen ein traditionelles Objekt von Kritik. Aber sie formen noch weitere gesellschaftliche Konflikte. Die aus dem organisationalen Dual von Struktur und Subjekt (Wendt 2020) resultierende Spannung der Arbeitsteilung erzeugt systematisch Formen entfremdeter Arbeit (Marx 1974), die bis heute als Kontrastfolie für Diskurse ihrer Humanisierung dienen. Die Organisation aber allein als Herrschaftszusammenhang zu verstehen (Türk 1995), marginalisiert deren komplexe Eigenlogik, um gesellschaftliche Gegensätze leichter politisieren zu können. Gesellschaftliche Verhältnisse nur als Produktionsverhältnisse zu politisieren, übersieht, dass es sich regelmäßig um Organisationsverhältnisse handelt, die sich nicht auf ökonomische Motive reduzieren lassen. Eine Auflösung dieser Grundkonflikte mittels partizipativer Demokratisierung der Organisation kann nicht gelingen, obwohl entsprechende Ideen der Organisationsgestaltung bereits seit dem Beginn der Managementlehre verhandelt werden (Wendt 2020). Der Gegensatz von organisationalen Erwartungen und subjektiven Ansprüchen ist untrennbar mit dem Prinzip der Organisation verknüpft. Mit der Abflachung von Hierarchien, der Demokratisierung von Kompetenzen und neuen Formen der Arbeitsorganisation verschwindet die Kritik an einer Entfremdung der Arbeit und der bürokratischen Gängelung von Mitarbeitern nicht. Dass individuelle Unterschiede in partizipativen Settings immer wichtiger werden (Manhart et al. 2020), ändert daran nichts. Die vergrößerten Handlungsspielräume der Mitarbeiter implizieren ein höheres Maß an Verantwortung und Selbstorganisation, was gern als fortschreitende Verunternehmerung kritisiert wird (Pongratz und Voß 1988; Bröckling 2007). Auch hier wird die strukturelle Komplexität der Organisation zugunsten einer eingängigen Kritik marginalisiert.

\section{Populismus als Diskursmuster der Moderne}

Trotz einer langen Vorgeschichte hat es sich erst in der Gegenwart etabliert, bestimmte Positionen als ,populistisch“ zu bezeichnen. Der eigentlich pejorativ gemeinte Begriff wird von den so bezeichneten Gruppen und Personen aber zunehmend als positive Selbstbezeichnung übernommen (Mouffe 2018). Populismus ist längst ein politischer Kampfbegriff, so dass selten geklärt wird, was genau denn nun das Populistische an einer inkriminierten Position sei. Kritiker des Populismus verstehen ihn regelmäßig als wesentlich politisches Phänomen, so z. B. wenn er als Reaktion auf Probleme der demokratischen Regierungsform und des Rechtsstaats dargestellt wird (Wielenga und Hartleb 2011; Priester 2012; Rosanvallon 2016; Decker 2017). Populisten greifen dies gern auf, wenn sie sich als Kritiker des Parlamentarismus oder 
auch der Gewaltenteilung im Namen eines Volkes gerieren, das von den Verteidigern leerer politischer Formen längst vergessen worden sei.

In diesem Diskurs aktualisieren sich, zumeist unbemerkt, Elemente aus der traditionellen Lehre von den Regierungsformen. Im deutschsprachigen Raum wurde sie zuletzt von Wilhelm Roscher zum Verständnis seiner eigenen Gegenwart systematisch verwendet (Roscher 1892; Manhart 2011, S. 223 f.). Unter anderem zur Erklärung der faktischen Selbstabschaffung der zweiten französischen Republik mithilfe demokratischer Referenden, die sowohl die Machtübernahme von Louis Napoleon Bonaparte wie auch dessen spätere Annahme des Kaisertitels legitimierten, griff Roscher auf die antike Lehre vom Kreislauf der Regierungsformen (Koselleck 1989, S. 70 f.) zurück. Die Geschichte der modernen Demokratie schien daher für Roscher schon wieder zu Ende, bevor sie so richtig begann. Sein Zeitgenosse Karl Marx zeigte hingegen in seiner berühmten Analyse derselben Ereignisse aus dem Jahr 1852 („Der achtzehnte Brumaire des Louis Bonaparte“) kein positives Interesse an irgendwelchen Formen des Politischen (Marx 1989). Ausgerichtet auf eine fundamentale Änderung gesellschaftlicher Verhältnisse konnte es nicht ernsthaft auf das politisch-bürokratische Klein-Klein einer Regierungsformenlehre, auf die letztlich bloß formalen, von ganz anderen Interessen getriebenen Händel der öffentlichen Meinungsbildung und Entscheidungsfindung in einem Staatswesen ankommen. Die soziale Frage und die für Marx einzig akzeptable Antwort hierauf, die soziale Revolution, haben ökonomische Ursachen, keine politischen. In diesem Sinne ist Marx zeitlebens ein ganz unpolitischer Denker geblieben, auch wenn man natürlich dieses Denken gegen die Politik, an dessen vielfältigen politischen Wirkungen ja kein Zweifel besteht, selber als politisch auffassen kann. Letztendlich ist sein Denken aber eine Form des ökonomischen Fundamentalismus.

Bei aller oberflächlichen Ähnlichkeit in der Ablehnung, bzw. dem oft zur Schau gestellten Desinteresse an politischen Formen, ist Populismus aber eben kein Fundamentalismus, schon gar kein Volksfundamentalismus (Populismus), was immer das sein könnte. Ein genuin politisches Verständnis von Populismus, z. B. als Folgeerscheinung der Demokratie, versteht diesen nun geradezu traditionell. Schon bei Polybios und Aristoteles entartet die Demokratie bzw. Politie zwingend aus sich selbst heraus. Die sich zunehmend individualisierenden Interessenkonflikte in der Polis führen zu einer Blockade der Regierung bzw. einer Abkehr ihrer Maßnahmen vom Gemeinwohl, was die Monarchie als durchsetzungsstarke Einherrschaft wieder als erstrebenswert erscheinen lässt. Roscher knüpft hieran an und erkennt im Cäsarismus jene moderne Form des Übergangs zur Monarchie, in der eine charismatische Einzelperson, bzw. eine mit dieser Person verbundene Clique, die Bevölkerung dazu bringt, sich ihrer Rechte zugunsten eines starken Regierungshandelns freiwillig zu entledigen. Bei Roscher ist die Bewertung des Cäsarismus ambivalent, weil er aus der Natur einer auf dem Volkswillen aufruhenden Republik notwendig entspringt. Zwar vertritt wohl heute kaum jemand mehr die Vorstellung einer quasi gesetzmäBigen Abfolge der Regierungsformen, doch ähneln sich politische Auffassungen des Populismus und Roschers Herleitung des Cäsarismus durchaus, wenn z. B. beide wesentlich als Folge der Volksherrschaft, genauer der Partizipation des Volkes an dieser Herrschaft erscheinen, wobei Medienmanipulationen und anderen Formen der 
Beeinflussung der Bevölkerung eine entscheidende Bedeutung auf dem Weg in die Diktatur zukommt.

Es scheint also geradezu auf der Hand zu liegen, Populismus als ein genuin politisches Phänomen zu verstehen. Aber in der Leichtgängigkeit dieser Argumentation liegt auch eine Gefahr. Denn eine solche Auffassung ist selber politisch. Sie ist immer Partei, d.h. zumeist gegen den Populismus und seine politischen Folgen gerichtet. Sie droht deshalb, analytische und damit auch pädagogische Möglichkeiten zu verschenken, die zu dessen Verständnis beitragen können, soweit man nicht gleich, quasi als Gegengift, eine fatalistisch-tragische Haltung einnimmt (Runciman 2020). Den Populismus aber vor allem als Verfallsform der modernen Mediendemokratie und ihrer Abhängigkeit von einer manipulierbaren Wahlbevölkerung aufzufassen, befördert leicht eine unangenehme soziale Schieflage der Beurteilung bzw. eine politisch-elitäre Beobachterposition. Sie impliziert vor allem eine Zwangsläufigkeit, wie sie sich schon in den Kreislauflehren der Regierungsformen findet. Dies aber kann einer angemessenen Reaktion der Erwachsenenbildung nicht zuträglich sein. Versteht man Populismus nicht als ,ursprünglich“ politisch, so lässt er sich als Reaktion auf die organisierte Komplexitätssteigerung einer zunehmend dezentralisierten, heterarchischen Moderne betrachten, die gerade deshalb auf eine Vielfalt sektoral begrenzt agierender Organisationen angewiesen ist. Folgerichtig kann auch die politische Antwort auf den Populismus nur in einer weiteren Steigerung der Komplexität der Demokratie bestehen (Rosanvallon 2017), denn mit weniger an Organisation wird man nicht rechnen dürfen. Erwachsenenbildung und Organisationspädagogik können hierfür an die Tradition einer Beförderung von Bildung als Komplexitätsfähigkeit anknüpfen (Diendorfer und Sandner 2017; Manhart 2018; Manhart und Wendt 2020).

Eine gesellschaftstheoretische Erweiterung der Perspektive auf den Populismus würde es hingegen erlauben, der Frage nachzugehen, ob Populismus nicht eigentlich eine Ablehnung des Politischen impliziert, also eine Unpolitik ist, die nur unter anderem auch in der politischen Arena zelebriert wird. Versteht man das Politische als eine spezifisch regulierte Art der Meinungsbildung und des Meinungskampfes (Arendt 1994, S. 354 ff.) bzw. als ein - in bestimmter Weise reguliertes - Subsystem der Gesellschaft, das kollektiv bindende Entscheidungen verfahrensförmig produziert (Luhmann 2000a, S. 69 ff.), so wird erkennbar, worauf dessen Ablehnung beruht. Mit mehr Distanz zum Politischen kann man es sich auch leichter offenhalten, zwischen Populismus und den Populisten zu unterscheiden. Führende Populisten sind nicht selten politische Machiavellisten. Ihr Machtstreben und Politikverständnis ist letztlich ganz traditionell (Sternberger 1984, S. 159ff.) und sollte nicht mit ihrer Fremd- oder Selbststilisierung als Populisten verwechselt werden. Denn dieses Verständnis ist für eine erwachsenenbildnerische Intervention der entscheidende Ansatzpunkt. Das Problem ist nicht, dass man Donald Trump, Jair Bolsonaro, oder Rodrigo Duterte pädagogisch kaum erreichen wird, sondern, dass man mit einer Ableitung des Populismus aus dem Verhalten und den Aussagen dieser Personen deren Machtstrategien folgt. An der pädagogisch relevanten populistischen Einstellung geht das vorbei. Dass Machtpolitiker den Populismus für ihre Zwecke nutzen, sollte nicht dazu verleiten, die populistische Perspektive mit dem Machtkalkül und dem damit verbundenen politisch-strategischen Formenbewusstsein genau dieser Politiker 
gleichzusetzen. Das Gegenteil dürfte der Fall sein. Populisten sind an den Formen und Verfahren des Politischen ebenso desinteressiert wie an der Macht. Es handelt sich weniger um Politik- als um Organisationsverdrossenheit. Der Ruf nach mehr Kontrolle (,get back control“) meint also gerade nicht mehr Staat, mehr Verwaltung und mehr Organisation. Eine Gleichsetzung populistischer Haltungen mit Arendts ganz anders begründetem Misstrauen gegenüber den notwendig unpolitischen „Verwaltungstalente(n) “ politischer Parteien (Arendt 1994, S. 353) oder mit Marx' Kritik der politischen Ökonomie als ökonomisierter Politik ginge ebenso an grundlegenden Unterschieden vorbei, wie die übliche Vermengung mit den Anhängern von Verschwörungstheorien oder religiösen wie völkischen Fundamentalisten.

\section{Populismus und Fundamentalismus - zwei gegensätzliche Formen der Komplexitätsreduktion}

Für ein besseres Verständnis des Populismus ist es notwendig, diesen vom Fundamentalismus und von Verschwörungstheorien zu unterscheiden. Diese Differenzierung kann der Erwachsenenbildung helfen, zielgenaue, adressatenorientierte Strategien für die pädagogische Auseinandersetzung zu entwickeln (Möllers und Merzel 2018; Buchberger und Mittnik 2019; Deichmann und May 2019). Denn gegenüber Fundamentalisten und Verschwörungstheoretikern kommt es auf ganz andere pädagogische Vorgehensweisen an als gegenüber Populisten. Zugleich verliert die Erwachsenenbildung pädagogische Möglichkeiten, wenn sie Populismus allein als politisch motivierte Kommunikationsform versteht. Populismus findet sich, wie der Fundamentalismus, in allen Bereichen der Gesellschaft und kann daher auch in all diesen pädagogisch bearbeitet werden.

Fundamentalismus wie Populismus sind jeweils eigenständige Reaktionen auf spezifische Strukturbildungen der Moderne und sie begleiten diese seit ihrer Entstehung. Beiderlei Kritik entzündet sich an der heterarchischen Struktur der modernen Gesellschaft, die mit der Ausdifferenzierung von Subsystemen nicht nur auf darin institutionalisierte Verfahren, sondern auch auf eine Vielzahl von Organisationen angewiesen ist. Deren Kontroll- und Steuerungsambitionen treten in Form rationaler Planungen auf, die den tatsächlichen Vollzügen regelmäßig nicht entsprechen. Auf das Steuerungsproblem von Organisationen, wie die strukturell angelegte Unmöglichkeit, aus dem einen Subsystem in ein anderes kontrolliert hineinzuagieren, reagiert der Fundamentalismus aber ganz anders als der Populismus.

\subsection{Fundamentalismus}

Im Unterschied zum Populismus kritisieren Fundamentalismen Organisationen und soziale Regelgeflechte nicht an sich, vielmehr nutzen sie diese explizit für ihre Zwecke und sie vertreten regelmäßig überaus rigide soziale Regelungsansprüche. Fundamentalismen reduzieren die Komplexität der modernen Gesellschaft, indem sie eines ihrer Subsysteme für zentral bzw. fundamental erklären. Dessen Dynamik und Logik gilt als für andere soziale Felder maßgebend. So ist der religiöse Fundamentalismus durch den Anspruch gekennzeichnet, die gesamte Gesellschaft habe in all ihren Voll- 
zügen den geoffenbarten Lehren dieses oder jenes religiösen Schrifttums zu folgen. Die Regeln der Religion sollen die Regeln der Gesellschaft sein, weil es der Wille Gottes ist. Nicht anders, nur unter Ersetzung Gottes, argumentieren ökonomische Fundamentalisten, für die z.B. der Markt oder die fallende Profitrate das Leben aller bestimmen soll. Gleiches gilt für einen wissenschaftlichen Fundamentalismus, der alle sozialen Verhältnisse nach den Regeln und Erkenntnissen der Wissenschaft auszurichten gedenkt. Fundamentalismen negieren immer gesellschaftliche Binnendifferenzierungen zugunsten der Kommunikations- und Handlungslogik eines Teilsystems. Dies geschieht im Namen des Guten, sei dies nun Gott, der Markt, die wissenschaftliche Wahrheit, Gesundheit oder auch, wie im Rassismus, das Wohlergehen einer Rasse. Hieraus entspringen verschiedene fundamentalistische Politiken, also Ansprüche und Vorgehensweisen, die aus der Perspektive eines Subsystems, wie z. B. der Religion, auf die Politik ausgreifen. Diese unterschiedlich fundamentalisierten Politiken sind von einem politischen Fundamentalismus zu unterscheiden. In diesem soll wiederum die Politik, ohne Rücksichtnahme auf die Eigenlogiken anderer gesellschaftlicher Bereiche, diese steuern. Insbesondere Diktaturen und die sozialistischen Planwirtschaften der Nachkriegszeit können als typische Beispiele für eine entsprechende Praxis gelten (Koenen 2010), wobei letztere lieber als wissenschaftlicher Fundamentalismus (,Historischer Materialismus“) gesehen werden wollten. Marx selbst hingegen, dies wurde schon erwähnt, ist, bei aller inhaltlichen Differenzierung im Detail, letztlich ein ökonomischer Fundamentalist. Marx' Theorie enthält kein politisches Konzept, das über die Revolution der Gesellschaft, d.h. die Abschaffung jeder bekannten Politik hinausgeht. Mit Populismus haben all diese Positionen aber nichts zu tun.

\subsection{Populismus}

Im Gegensatz zum Fundamentalismus verweigert es der Populismus systematisch, die fundamentale Stellung irgendeines Subsystems anzuerkennen. Weder funktionale Grenzziehungen noch Organisationen werden als Regelungszusammenhänge akzeptiert. Kern des Populismus ist die Behauptung, es könne, wenn man nur wolle, alles ganz einfach sein. Einfach meint hier vor allem, Willensakte könnten ohne langwierige Regulierungen und ohne Verwässerung bzw. Sinndiffusion in der organisierten Umsetzung verwirklicht werden. Im Populismus drückt sich ein tiefsitzender Widerstand gegen die der Moderne inhärente Spannung aus, dass zwar das Neue und Offene beständig propagiert, in planmäßigen Reformen aber vor allem das Alte in Organisationen reguliert wird. Die für die Moderne typische Dauerreform der Gesellschaft verlängert die Gegenwart mittels organisationaler Planungen in eine Zukunft, die als unbekannt zwar unbegrenzt positiv aufgeladen werden kann (Esposito 2005), deren praktische Umsetzung durch Organisationen aber gerade deshalb als systematische Verhinderung des Richtigen und damit als Zumutung empfunden wird. Organisationen agieren im Kontext aller Subsysteme ohne Abstimmung aufeinander und sind damit zugleich Motoren und Adressen populistischer Kritik. Da institutionelle Regelgeflechte und Subsystemgrenzen die gesamte Gesellschaft durchziehen, ist populistische Kommunikation auch überall zu finden. Man unter- 
schätzt daher die Reichweite und Evidenz populistischer Kritik, versteht man sie vorrangig politisch.

Populistische Kritik richtet sich gegen das Wissenschafts-, Wirtschafts- oder Rechtssystem ebenso wie gegen Maßnahmen von Gerichten, Unternehmen, Krankenhausgesellschaften oder Einrichtungen der öffentlichen Verwaltung. Selbstverständlich gibt es auch einen pädagogischen Populismus, dessen Kritik an Schule und Lehrern vor allem das Technologiedefizit der Erziehung, also die Grenze zwischen Sozialwelt und Psyche, zwischen Kommunikation und Denken, ignoriert. Deshalb kann er behaupten, man müsste Kinder nur richtig, also hierzu und dazu erziehen wollen, dann würde das auch klappen. Konkrete pädagogische Organisationen werden als bürokratische Verhinderungsanstalten kritisiert. Populistische Behauptungen, die jede Grenze zwischen dem Erziehungssystem, den Möglichkeiten von Schule, Heimerziehung und Universitäten und den anderen Subsystemen der Gesellschaft ignorieren, sind Legion. Sie sollten allerdings nicht mit dem pädagogischen Fundamentalismus eines Johann Gottlieb Fichte verwechselt werden. Für Fichte kam es in nationalpolitischer Absicht darauf an, den bloß individuellen Willen des Einzelnen in der „Bildungsfabrik“ des Staates sorgfältig zu brechen, damit er in der ungeteilten Einheit der Deutschen Nation endlich aufgehen könne (Fichte 1971, S. 589; Manhart 2011, S. 446-457). Pädagogischen Populisten geht es hingegen nicht um eine fundamentale Rolle der Pädagogik, schon gar nicht um den Staat als Erziehungsanstalt, vielmehr stören sie sich an ihrer inhaltlichen und verfahrenslogischen Komplexität.

Dass populistische Kritik vor allem im politischen System besonders auffällt, hat viel mit der Aufmerksamkeitslenkung durch das System der Massenmedien zu tun, das nicht nur über Kritik an Staat, Verwaltung und Parteien vorrangig berichtet, sondern populistische Einlassungen auf diese Weise vielfach erst politisiert. Digitale Netzwerke sorgen für eine soziale wie thematische Reichweitenvergrößerung populistischer Kritik. Als politische Richtung ist Populismus im Gegensatz zu den Spielarten subsystemspezifischer Fundamentalismen durch die explizite oder implizite Behauptung gekennzeichnet, dass Politik nicht in allen, sondern als alle anderen Subsysteme der Gesellschaft entscheiden könne. Es geht also um die Auflösung von Politik, indem diese mit den Logiken der anderen gesellschaftlichen Subsysteme bis zur Unkenntlichkeit fusioniert. Insofern ist dieses Denken tatsächlich unpolitisch, denn eine eigenständige Sphäre des Politischen, erst recht eine Eigenlogik der Politik, ist genau das, was kritisiert und ignoriert wird. Es handelt sich beim politischen Populismus also nicht um einen Politikfundamentalismus, wie er in einer Vielzahl von Diktaturen Praxis ist. In diesen ersetzen, zumindest dem Anspruch nach, politische Entscheidungen wirtschaftliche, religiöse, juristische oder auch wissenschaftliche Abläufe. Die Ausweitung der Möglichkeiten des Entscheidens sollen hingegen im Populismus nicht durch das und zu den spezifischen Bedingungen der Politik geschehen, vielmehr wird ,politische“ Wirksamkeit gerade als deren Aufhebung im Sinne der Abschaffung regulierter Foren der Meinungsbildung, formaler Abläufe und gewaltenteilender Begrenzungen verstanden. Dass dies in der Praxis gerade auf einen starken Staat bzw. eine Diktatur hinauslaufen kann, liegt in der Logik der organisierten Moderne, ändert aber nichts daran, dass Populismus dies nicht intendiert. Sein Kern ist eine regelaverse Delegitimierung von Verfahren, die das 
politische System und seine Organisationen (Staat, Parteien, Verbände) relativiert und nicht etwa deren Strukturen fundamentalisiert.

Dieser antiformalistische Zug des Populismus gilt konsequenterweise nur formal, ist also von jeder dauerhaften inhaltlichen Festlegung, Verfahrensweise oder normativen Bindung, z. B. an das Gemeinwohl oder gar an konkrete Vorstellungen des Politischen oder des Staates, unabhängig. Es soll einfach richtig und endlich auch mal wirklich entschieden werden. „Wirklich zu Entscheiden“ bedeutet, den Unterschied zwischen Entscheidung und Umsetzung zu negieren. Organisationen, Staat und Parteien, aber auch Medien, werden daher vor allem als diese Umsetzung korrumpierende Entscheidungsverhinderer aufgefasst. Richtig ist, was der ,gesunde Menschenverstand“, also man selber im Moment dafür hält. Aus diesem Verständnis entspringen weniger individualistische, als vor allem subjektiv leicht verfügbare Positionen, an denen man, allerdings nur für kurze Zeit, normativ vehement, also nicht lernbereit, festhält (Luhmann 1975b, S. 55 ff.). Populistische Positionen aktualisieren die Konditionen eines Achtungsmarktes (Luhmann 1990b), wenn moralische Kommunikation an die Stelle inhaltlicher Argumente tritt. Indem man Achtungsbedingungen kommuniziert, kann auf inhaltliche Begründungen verzichtet werden. Denn ein dauerhaftes Festhalten an konkreten Positionen würde Verfahren der Abstimmung und Konsistenzprüfung erfordern, die gemieden werden. Deshalb werden auch allgemeine Werte zugunsten subjektiver Befindlichkeiten suspendiert. Dabei wird nicht moralisierende Kommunikation abgelehnt, im Gegenteil, aber die Absicherung moralischer Urteile erfolgt nicht über das Aushandeln relevanter Werte, sondern durch die Ausflaggung individueller Befindlichkeiten. Im Gegensatz zur Stabilisierung von Werten im wechselseitigen Meinungsaustausch können Befindlichkeiten vom subjektiven Gefühl individueller Betroffenheit abhängig gemacht werden (Frevert 2019). Für den Populismus ist diese Verweigerung gegenüber einer öffentlichen Wertebindung zentral. Der Rekurs auf intransparente Befindlichkeiten erlaubt es, aktuelle Dringlichkeit mit dem nötigen Spielraum zu verbinden, sich morgen anders fühlen zu können.

Es geht populistischen Einlassungen also grundsätzlich nicht darum, in einem wechselseitigen Austausch die eigene Position zu vertreten, sie entlang allgemein geteilter Prinzipien oder gar in Verfahren zu legitimieren, sie zu korrigieren oder wenigstens argumentativ zu härten. Das würde bedeuten, die Annahme und Umsetzung zumindest teilweise anderen überlassen zu müssen, was auf Regeln und Verfahren hinausliefe und daher als problematisch erscheint. Für den Populismus würde Hannah Arendt wohl schon deshalb die Fähigkeit zur politischen Meinungsbildung in Zweifel ziehen, denn für sie formt erst der Austausch mit Anderen einen Gedanken, einen Einfall zu einer Meinung um. Meinungen sind für Arendt immer öffentlich und der Austausch über und das Ringen um das überzeugendere Argument sind daher auch der prozessuale Kern des Politischen (Arendt 1994, S. 357 ff). Populismus ist hingegen an Meinungsbildungsprozessen dieser Art ebenso wenig interessiert wie an jeglicher Form von Verfahrensförmigkeit. 


\subsection{Der Unterschied zwischen Populismus und Verschwörungstheorien}

Das bedeutet auch, dass Vertreterinnen und Vertreter von Hyperrationalisierungen, wie sie für Verschwörungstheorien kennzeichnend sind, zwar personell gerne an populistische Bewegungen andocken, die Unterschiede aber ebenfalls grundsätzlicher Natur sind. Populistische Bewegungen haben wegen ihrer amorphen Binnenstruktur Verschwörungstheorien zwar wenig entgegenzusetzen, so dass Populisten diesen fallweise durchaus zustimmen. Ihre Konzepte funktionieren aber ganz anders. Populismus marginalisiert die Bedeutung von Verfahren, Grenzen und Regeln. Populisten sind daher offen dafür, Zufälle und Inkonsistenzen beim Vertreten ihrer Anliegen, aber auch in der Gesellschaft, hinzunehmen. Verschwörungstheoretiker hingegen nehmen gerade Zufälle und Inkonsistenzen zum Anlass, diese systematisch, auf dahinterliegende Absichten und Regeln hin, zu rationalisieren, also in eine Ordnung zu bringen. Die Aversion gegen Zufälle geht bei ihnen so weit, dass sie sich gesellschaftliche Prozesse nur als Produkte gelingender menschlicher Absichten und in der Folge dann auch als hierarchisch organisiert vorstellen können. Die Unübersichtlichkeit der Moderne wird konsequent als wiederum beabsichtigter Trick verstanden, der dazu dient, die eigentlich durchkontrollierte Wirklichkeit zu verschleiern (,der tiefe Staat“, etc.). Das Gegebene nicht so zu nehmen, wie es ist, ist der Startpunkt jeder Rationalisierung (Manhart 2011, S. 22 ff.). Insofern folgen Verschwörungstheoretiker einer geradezu hypertrophen Rationalität. Sie sind Rationalitätsfundamentalisten, die sich nicht nur zum Nachweis ihrer Thesen auf durchaus komplexe Methoden einlassen, sondern auch an eine Elite vollrationaler Personen glauben, zu der sie natürlich vor allem selbst gehören.

Personalisierungen haben also im Fundamentalismus wie in Verschwörungstheorien einen ganz anderen Hintergrund als im Populismus. Da Subsysteme keine Akteure sein können, Organisationen aber wegen ihrer Regelgeleitetheit abgelehnt werden, läuft dies auch im Populismus auf eine Zentralstellung von Personen als Entscheider hinaus. Entscheidungen werden konsequenterweise als personeninterne Prozesse, nicht als soziale Formen verstanden. Diese faktische Tendenz zur Personalisierung ist, ebenso wie die politische Möglichkeit einer Diktatur, aber lediglich ein sekundärer Effekt der grundlegenden Skepsis gegenüber strukturellen Grenzen und organisierten Verfahren. Vor allem läuft dies nicht zwingend auf bestimmte Personen hinaus. Dass machtorientierte, charismatische Persönlichkeiten diese Konstellation politisch für sich nutzen, sollte nicht dazu verleiten, diese Personen und deren Behauptungen in der Analyse des Populismus für zentral zu halten. Populismus ist eine verbreitete Reaktion auf die komplexe, dezentralisiert-heterarchische Struktur der Moderne, dem gerade die fixe Kopplung sozialer Zusammenhänge in Form institutionalisierter Regeln und Organisationen und deren daraus entspringenden raumzeitlichen Begrenzungen Anlässe zur Kritik liefern. Er wendet sich von bestimmten Personen deshalb genauso leicht wieder ab, wie er sich ihnen zugewandt hat. 


\section{Organisation und Partizipation}

Da Organisationen für die Persistenz und Durchschlagskraft des Populismus eine zentrale Rolle spielen, lassen politische Konzepte einer Demokratisierung der Demokratie (Manow 2018) die Schwierigkeiten und Handlungsdilemmata von partizipationsorientierten Gegenstrategien gut erkennen. Analoge Beispiele und Probleme finden sich, der Verbreitung von Organisationen und populistischen Kritiken entsprechend, auch in anderen gesellschaftlichen Feldern, man denke an die Einführung flacher Hierarchien in Unternehmen und Verwaltungen, an Konzepte von New Work oder an Citizen-Science-Projekte in der Wissenschaft. Regelmäßig geht es um eine Demokratisierung von Organisationen bzw. um einen Auf- und Umbau von Regelgeflechten mittels partizipativer Verfahren (Manhart et al. 2020). Doch verstärkte Partizipation und individuelle Teilhabemöglichkeiten sind ohne eine weitere Steigerung organisierter Komplexität nicht zu haben. Auf organisationsbedingte Probleme wird mit einem Mehr an Organisation reagiert, und das heißt, dass auf Probleme wachsender Komplexität ihre Steigerung folgt. Dementsprechend spricht auch Rosanvallon von einer Verkomplizierung der Demokratie durch neue Beteiligungsstrategien als Antwort auf ihre Krise (Rosanvallon 2017). Es kommt deshalb pädagogisch auch darauf an, hier nicht zu viel zu versprechen und immer wieder zu vermitteln, warum das so ist.

Das Ziel, organisational verfertigte Einschränkungen und Begrenzungen durch Partizipation aufzubrechen, ist eng mit der Vorstellung verknüpft, das Ende der Hierarchie und der exkludierenden Wirkung von Zuständigkeiten ermögliche eine Befriedung der Organisation und ihrer strukturellen Spannungen. Faktisch spielen sich aber verfahrensförmige Abläufe sowie die daraus resultierende unzureichende Berücksichtigung subjektiver Bedarfslagen erneut ein. Partizipation muss daher im Kontext organisationaler Handlungsimperative und Strukturvorgaben verstanden und weiterentwickelt werden. Das Spannungsfeld zwischen personenunabhängiger Strukturfestlegung und personenabhängiger Partizipation kann nicht aufgelöst, die Strukturlogik von Organisationen - hierarchisch-horizontale Koordination und spezifizierend-exklusive Kompetenzverteilung - nicht einfach ersetzt werden. Denn ohne eine geregelte Erzeugung sozialer Zusammenhänge kann man auch nicht nachhaltig an ihnen partizipieren. Partizipation kann deshalb immer nur eine Beteiligung an immer schon vorstrukturierten Zusammenhängen sein. Überprüfbare Teilhabe kann es nur in Organisationen und nur als Organisation geben. Mehr Partizipation setzt deshalb eine Vermehrung von Regeln und eine Ausweitung organisierter Sozialität voraus. Diese, im Blick auf populistische Kritik, geradezu dilemmatische Konstellation lässt sich nicht ändern, aber man kann sie praktisch vorführen und erklären.

Die pädagogische Aufgabe besteht darin, hierauf mit der Vermittlung eines realistischen Organisations- und Partizipationsverständnisses vorzubereiten. Das Versprechen einer regelfernen Freiheit hilft nicht weiter. Partizipation erfordert immer Formen wechselseitiger Abstimmung, die für den Einzelnen eine andauernde Herausforderung sind. Organisierte Regelgeflechte erfordern deshalb Wissen und Fähigkeiten im Umgang mit Komplexität, die erlernt werden müssen. Man muss gegenüber Partizipation nicht so skeptisch urteilen wie Luhmann (Luhmann 1990a, S. 164), aber unbestreitbar werden Personengruppen, die z. B. von Gefahren betrof- 
fen sind, mittels Partizipation zu Riskonehmern, d.h. sie entscheiden nun mit und können nicht mehr so einfach Widerstand gegen eine organisierte Gefährdung leisten. Dies mag man als zu kritisierende Entlastungsstrategie von Organisationen und Entscheidern verstehen, pädagogisch geht es aber um die Befähigung zur Verantwortungsübernahme, was deren wirksame Ablehnung miteinschließt. Die Erwartung, durch Partizipation vermeintliche Gegensätze, wie jenen von selbstbestimmter Teilhabe und fremdbestimmter Regelung, von Freiheit und Zwang aufzuheben, kann nur enttäuscht werden. Es kommt daher darauf an, diese Gegensätze an einem komplexeren Organisations- und Gesellschaftsverständnis zu relativieren, womit nicht nur das Enttäuschungspotential, sondern auch kognitive wie emotionale Verhärtungen verringert werden, die die individuelle Basis populistischer Kritik sind.

Die Eigenlogik partizipativer Prozesse vor dem Hintergrund der Dialektik der Organisation und ihrer komplexen Dynamik zu reflektieren, arbeitet zudem mit an einem anderen pädagogischen Aspekt von Partizipation. Klassisch bezeichnet der Begriff der Bildung jene Fähigkeit, mit der eigenen und fremden Komplexität produktiv umzugehen (Manhart und Wendt 2020). Soziale Komplexität wird nun in partizipativen Verfahren durch die Intransparenz und Zufallsabhängigkeit von Kommunikations- und Interaktionsprozessen systematisch erzeugt (Manhart und Wendt 2019; Wendt 2020). Partizipative Prozesse fungieren deshalb nicht nur als Ventil und Projektionsfläche für komplexitätsreduzierende Deutungen und Kritik, sondern sie sind auch zentrale soziale Voraussetzung individueller Bildung. Partizipative Gestaltung von Organisationsstrukturen setzt einen produktiven Umgang mit der von den partizipierenden Individuen erzeugten Komplexität voraus (Schröer und Wendt 2018). Vor dem Hintergrund - der nicht zuletzt durch Prozesse der Digitalisierung - immer besser gelingenden Strukturautomation (Wendt 2020; Wendt und Manhart 2020), sind Organisationen für die Fortsetzung der eigenen Dynamik immer stärker darauf angewiesen, individuelle Bildung durch komplexe Arrangements produktiver Widerstände zu ermöglichen.

Eine populistische Fassung von Partizipation entleert hingegen den Begriff. Partizipation wird dann vor allem als Abbau widerständiger Regeln verstanden. Man wird gehört, gesehen, wahrgenommen - endlich. Partizipation wird hier als diffuses, keinerlei Vorgaben oder Formen akzeptierendes (Mit-)Machen vorgestellt. Konkrete Ausführungsbedingungen, jenseits der bloßen Möglichkeit eines partizipativ-emotionalen Jetzt, spielen keine Rolle. Inhaltliche oder formale Bedingungen implizieren Festlegungen, die zu vermeiden der Kern dieser Imagination von Teilhabe ist. Das gilt nicht nur für politisch rechts verankerte Zugehörigkeitskonzepte wie die Volksgemeinschaft, sondern auch für „das Volk“, den „,kleinen Mann“ oder Gegenbegriffe, wie ,die da oben“. All diese Formen der Partizipation entsprechen der regelaversen, antiorganisatorischen, sozial-formale Handlungsbegrenzungen negierenden populistischen Haltung, der man nicht mit falschen Versprechungen und Vereinfachungen entgegenkommen sollte. Durch Hierarchieabbau und die Vermehrung von Beteiligungsmöglichkeiten werden Organisationen nicht zu offenen Arenen demokratischer Partizipation und individueller Gleichberechtigung. Sie werden selbst zu gebildeten kollektiven Akteuren, indem sie individuelle Bildung am eigenen komplexen Strukturaufbau ermöglichen. Diese Pädagogik der Organisation besser zu verstehen, ist die noch einzulösende Voraussetzung dafür, sie als Organisationspädagogik produk- 
tiv für ein anderes (Selbst-)Verständnis von Organisation wenden zu können. Das könnte auch Organisationen und soziale Regelgeflechte wieder populärer machen, gerade weil sie komplex sind.

Open Access Dieser Artikel wird unter der Creative Commons Namensnennung 4.0 International Lizenz veröffentlicht, welche die Nutzung, Vervielfältigung, Bearbeitung, Verbreitung und Wiedergabe in jeglichem Medium und Format erlaubt, sofern Sie den/die ursprünglichen Autor(en) und die Quelle ordnungsgemäß nennen, einen Link zur Creative Commons Lizenz beifügen und angeben, ob Änderungen vorgenommen wurden.

Die in diesem Artikel enthaltenen Bilder und sonstiges Drittmaterial unterliegen ebenfalls der genannten Creative Commons Lizenz, sofern sich aus der Abbildungslegende nichts anderes ergibt. Sofern das betreffende Material nicht unter der genannten Creative Commons Lizenz steht und die betreffende Handlung nicht nach gesetzlichen Vorschriften erlaubt ist, ist für die oben aufgeführten Weiterverwendungen des Materials die Einwilligung des jeweiligen Rechteinhabers einzuholen.

Weitere Details zur Lizenz entnehmen Sie bitte der Lizenzinformation auf http://creativecommons.org/ licenses/by/4.0/deed.de.

\section{Literatur}

Arendt, H. (1994). Über die Revolution (4. Aufl.). München, Zürich: Piper.

Baecker, D. (2001). Der Witz der Organisation. In F. Dievernich (Hrsg.), Kommunikationsausbrüche. Vom Witz und Humor der Organisation (S. 221-232). Konstanz: UVK.

Bröckling, U. (2007). Das unternehmerische Selbst. Soziologie einer Subjektivierungsform. Frankfurt a.M.: Suhrkamp.

Buchberger, W., \& Mittnik, P. (Hrsg.). (2019). Herausforderung Populismus. Multidisziplinäre Zugänge für die politische Bildung. Schwalbach am Taunus: Wochenschau.

Decker, F. (2017). Populismus in Westeuropa. Theoretische Einordnung und vergleichende Perspektiven. In G. Diendorfer \& G. Sandner (Hrsg.), Populismus - Gleichheit - Differenz. Schwalbach a.T.: Wochenschau.

Deichmann, C., \& May, M. (Hrsg.). (2019). Orientierungen politischer Bildung im „postfaktischen Zeitalter". Wiesbaden: Springer.

Diendorfer, G., \& Sandner, G. (Hrsg.). (2017). Populismus - Gleichheit - Differenz. Schwalbach a.T.: Wochenschau.

Esposito, E. (2005). Geplante Neuheit: die Normalität der Reform. In G. Corsi \& E. Esposito (Hrsg.), Reform und Innovation in einer unstabilen Gesellschaft (S. 51-60). Stuttgart: Lucius \& Lucius.

Fichte, J. G. (1971). Zur Politik, Moral und Geschichte. Excurse zur Staatslehre. Politische Fragmente aus den Jahren 1807 und 1813, Bd. 7 (S. 574-589). Berlin: Mohr.

Frevert, U. (2019). Kapitalismus, Märkte und Moral. Wien: Residenz.

Hellmuth, T. (2019). Vergesst Populismus. Ein Essay über die Problematik eines diffusen Begriffs. In W. Buchberger \& P. Mittnik (Hrsg.), Herausforderung Populismus. Multidisziplinäre Zugänge für die politische Bildung. Schwalbach a.T.: Wochenschau.

Koenen, G. (2010). Was war der Kommunismus? Göttingen: Vandenhoeck \& Ruprecht.

Koselleck, R. (1989). Historische Kriterien des neuzeitlichen Revolutionsbegriffs. In R. Koselleck (Hrsg.), Vergangene Zukunft. Zur Semantik geschichtlicher Zeiten (S. 67-86). Frankfurt a.M.: Suhrkamp.

Lesch, W. (Hrsg.). (2017). Christentum und Populismus. Freiburg: Herder.

Luhmann, N. (1975a). Interaktion, Organisation, Gesellschaft. In N. Luhmann (Hrsg.), Soziologische Aufklärung 2. Aufsätze zur Theorie der Gesellschaft. (S. 9-20). Opladen: Westdeutscher Verlag.

Luhmann, N. (1975b). Die Weltgesellschaft. In N. Luhmann (Hrsg.), Soziologische Aufklärung 2 (S. 51-71). Opladen: Westdeutscher Verlag.

Luhmann, N. (1990a). Risiko und Gefahr. In N. Luhmann (Hrsg.), Soziologische Aufklärung 5 (S. 131-169). Opladen: Westdeutscher Verlag.

Luhmann, N. (1990b). Paradigm lost: Über die ethnische Reflexion der Moral. Rede von Niklas Luhmann anläßlich des Hegel-Preises 1989. Frankfurt a.M.: Suhrkamp. 
Luhmann, N. (1994). Die Gesellschaft und ihre Organisationen. In H.-U. Derlien, U. Gerhardt \& F. Scharpf (Hrsg.), Systemrationalität und Partialinteresse. Festschrift für Renate Mayntz (S. 189-201). BadenBaden: Nomos.

Luhmann, N. (1996). Die Realität der Massenmedien. Opladen: Westdeutscher Verlag.

Luhmann, N. (2000a). Die Politik der Gesellschaft. Frankfurt a.M.: Suhrkamp.

Luhmann, N. (2000b). Organisation und Entscheidung. Opladen: Westdeutscher Verlag.

Manhart, S. (2009). Der Preis der Freiheit. Bildung, Wissen, Organisation. Zeitschrift für Soziologie der Erziehung und Sozialisation (ZSE), 29(1), 80-95.

Manhart, S. (2011). In den Feldern des Wissens. Studiengang, Fach und disziplinäre Semantik in den Geschichts- und Staatswissenschaften (1780-1860). Würzburg: Königshausen und Neumann.

Manhart, S. (2018). Complex learning and the significance of measurement. In A. König \& J. Ravetz (Hrsg.), Sustainability science. Key issues (S. 296-317). London: Routledge.

Manhart, S., \& Wendt, T. (2019). Delokalisierung, Entzeitlichung und Entpersonalisierung organisierter Pädagogik. Zur digitalen Transformation organisationaler Raumzeit und ihres Subjekts. Zeitschrift für Weiterbildungsforschung (ZfW), 42(2), 235-248.

Manhart, S., \& Wendt, T. (2020). Bildung als Komplexitätsfähigkeit. In M. Oberlechner \& P. Duval (Hrsg.), Neue Konzepte des Humanismus für die Schule von morgen [Redefining humanism for schools of tomorrow. Redéfinir l'humanisme pour l'école de demain]. Frankfurt a.M.: Wochenschau Verlag.

Manhart, S., Wendt, T., \& Schröer, A. (2020). Individuelle Kreativität und organisierte Innovation. Elemente einer organisationspädagogischen Synthese. In C. Fahrenwald, N. Engel \& A. Schröer (Hrsg.), Organisation und Verantwortung. Jahrbuch der Sektion Organisationspädagogik (S. 339-353). Wiesbaden: Springer VS.

Manow, P. (2018). Die Politische Ökonomie des Populismus. Berlin: Suhrkamp.

Marx, K. (1974). Die entfremdete Arbeit. In K. Marx (Hrsg.), Ökonomisch-philosophische Manuskripte. Geschrieben von April bis August 1844. Nach der Handschrift (S. 149-166). Leipzig: Reclam.

Marx, K. (1989). Der achtzehnte Brumaire des Louis Bonaparte [1852]. In K. Marx \& F. Engels (Hrsg.), Ausgewählte Schriften in zwei Bänden (Bd. I, S. 267-371). Berlin: Dietz.

Möllers, L., \& Merzel, S. (Hrsg.). (2018). Populismus und Politische Bildung. Schriftenreihe der GPJE. Schwalbach a.T.: Wochenschau Verlag.

Mouffe, C. (2018). Für einen linken Populismus. Frankfurt a.M.: Suhrkamp.

Müller, M., \& Precht, J. (Hrsg.). (2019). Narrative des Populismus. Erzählmuster und Strukturen populistischer Politik. Wiesbaden: Springer VS.

Nassehi, A. (2011). Gesellschaft der Gegenwarten. Studien zur Theorie der modernen Gesellschaft II. Frankfurt a.M.: Suhrkamp.

Nassehi, A. (2017). Die letzte Stunde der Wahrheit/Kritik der komplexitätsvergessenen Vernunft. Hamburg: Sven Murmann.

Pongratz, H.-J., \& Voß, G. (1988). Der Arbeitskraftunternehmer. Eine neue Grundform der Ware Arbeitskraft? Kölner Zeitschrift für Soziologie und Sozialpsychologie, 50(1), 131-158.

Priester, K. (2012). Rechter und linker Populismus. Annäherungen an ein Chamäleon. Frankfurt a.M.: Campus.

Raphael, L. (2017). „Demokratie ist Geschichte“. Pierre Rosanvallons Untersuchungen zur Demokratie in praktischer Absicht. In: Soziopolis. https://soziopolis.de/beobachten/politik/artikel/demokratieist-geschichte/. Zugegriffen: 7. Juli 2020.

Rosanvallon, P. (2016). Die gute Regierung. Hamburg: Hamburger Edition.

Rosanvallon, P. (2017). Wider alle Vereinfachung. Zur Demokratie im 21. Jahrhundert. In: Soziopolis. https://soziopolis.de/beobachten/politik/artikel/wider-alle-vereinfachung/. Zugegriffen: 7. Juli 2020.

Roscher, W. (1892). Politik. Geschichtliche Naturlehre der Monarchie, Aristokratie und Demokratie. Stuttgart: Verlag der Gotta'schen Buchhandlung.

Runciman, D. (2020). So endet die Demokratie. Frankfurt a.M.: Campus.

Schröer, A., \& Wendt, T. (2018). Partizipationsorientierung als Forschungsstrategie der Organisationspädagogik. In M. Göhlich, A. Schröer \& S. M. Weber (Hrsg.), Handbuch Organisationspädagogik (S. 331-342). Wiesbaden: Springer.

Sternberger, D. (1984). Drei Wurzeln der Politik. Frankfurt a.M.: Suhrkamp.

Türk, K. (1995). „Die Organisation der Welt“. Herrschaft durch Organisation in der modernen Gesellschaft. Opladen: Westdeutscher Verlag.

Vehrkamp, R., \& Merkel, W. (2020). Populismusbarometer 2020. Populistische Einstellungen bei Wählern und Nichtwählern 2020. Gütersloh: Bertelsmann. Wissenschaftszentrum Berlin für Sozialforschung.

Wendt, T. (2016). Diesseits von Be- und Entschleunigung. Beratung als soziale Bearbeitung zeitlicher Komplexität. Sozial Extra, 40(4), 33-36. 
Wendt, T. (2019). Die moderne Suche nach Gelegenheiten. Plädoyer für eine zeitgemäße Beratung. Verhaltenstherapie und psychosoziale Praxis - VPP, 51(2), 293-301.

Wendt, T. (2020). Die nächste Organisation. Management auf dem Weg in die digitale Moderne. Bielefeld: transcript.

Wendt, T., \& Manhart, S. (2020). Digital Decision Making als Entscheidung, nicht zu entscheiden. Zur Zukunft des Entscheidens in der Digitalisierung. Arbeit, 29(2), 143-160.

Wielenga, F., \& Hartleb, F. (Hrsg.). (2011). Populismus in der modernen Demokratie. Die Niederlande und Deutschland im Vergleich. Münster: Waxmann.

Publisher's Note Springer Nature remains neutral with regard to jurisdictional claims in published maps and institutional affiliations. 